\title{
ON A GENERAL CLASS OF INTEGRALS OF THE FORM
}

$$
\int_{\mathbf{B Y}}^{\infty} \phi(t) g(x+t) d t^{*}
$$

\section{R. D. CARMICHAEL}

\section{INTRODUCTION}

In previous memoirs $\dagger$ I have considered the general theory of a class of infinite series of the form

$$
\Omega(x)=\sum_{n=0}^{\infty} c_{n} \frac{g(x+n)}{g(x)}
$$

where the coefficients $c_{0}, c_{1}, c_{2}, \cdots$ are independent of $x$ and where the function $g(x)$ is single-valued in a sector $V$ formed by two rays from zero to infinity and containing in its interior the positive axis of reals, is analytic at each point in this region for which $|x|$ is sufficiently large but finite, and has the asymptotic property expressed in the formula

$$
g(x) \sim x^{P(x)} e^{Q(x)}\left(1+\frac{a_{1}}{x}+\frac{a_{2}}{x^{2}}+\cdots\right) .
$$

Here $P(x)$ and $Q(x)$ are polynomials which we write in the form

$$
\begin{array}{ll}
P(x)=\mu_{0}+\mu_{1} x+\mu_{2} x^{2}+\cdots+\mu_{k} x^{k} & \left(\mu_{k} \neq 0 \text { if } k>0\right), \\
Q(x)=\alpha_{0}+\alpha_{1} x+\alpha_{2} x^{2}+\cdots+\alpha_{m} x^{m} \quad\left(\alpha_{m} \neq 0 \text { if } m>0\right) .
\end{array}
$$

(By $r^{8}$ we mean $e^{s \log r}$, where the principal determination of $\log r$ is taken.) In case $k=0$ we assume that $m>1$ in order to avoid a case which is unimportant so far as our present objects are concerned.

By means of these functions $g(x)$ we now introduce the general class of integrals

$$
J(x)=\int_{0}^{\infty} \phi(t) \frac{g(x+t)}{g(x)} d t,
$$

* Presented to the Society, April 13, 1918.

$\dagger$ These memoirs will be referred to by the numbers in the following list: I. These T $\mathrm{r}$ a $\mathrm{ns}$ actions, vol. 17 (1916), pp. 207-232. II. Bulletin of the American Mathematical Societ y, vol. 23 (1917), pp. 407-425. III. A merican Journal of Mathematics, vol. 39 (1917), pp. 385-403. IV. A merican Journal of $\mathrm{M}$ a the matics, vol. 40 (1918), pp. 113-126. 
where $\phi(t)$ is finite and single-valued for finite $t \geqq 0,|\phi(t)|$ has an upper bound in every finite interval $(0 \tau)$, and $\phi(t)$ is integrable in every such interval.* It is obvious that deep-lying analogies exist between series $\Omega(x)$ and integrals $J(x)$.

The most important case of integrals $J(x)$ which has figured in the literature is that for which we have

$$
g(x)=e^{-\frac{1}{3} x^{2}}, \quad \phi(t)=\psi(t) e^{\frac{1}{2} t^{2}} ;
$$

this gives rise to the Euler-Laplace integral $\dagger$

$$
I(x)=\int_{0}^{\infty} \psi(t) e^{-t x} d t
$$

the integral at the basis of the Laplace transformation. It is also a fundamental integral in Borel's integral definition of the sum of a divergent series.

Other special cases of particularly simple properties are suggested at once by the instances of series $\Omega(x)$ exhibited in Memoir II. One may consider also a generalization of integrals $J(x)$ analogous to the generalization $T(x)$ of the series $\Omega(x)$ given in Memoir II.

A sum of two particular integrals $J(x)$ also gives rise to an integral of interest. For the first of these integrals $J(x)$ we take

$$
\phi(t)=\frac{1}{2}[u(t)-i v(t)] e^{-\frac{1}{3} i\left(t^{2}-t\right)}, \quad g(x)=e^{-\frac{1}{2} i x(x-1)}, \quad i=\sqrt{-1},
$$

where $u(t)$ and $v(t)$ are real-valued functions of the real variable $t$ and $x$ is now to be restricted to real values. For the second of these we take functions $\phi$ and $g$ obtained from the foregoing on replacing $i$ by $-i$. Adding the two corresponding integrals $J(x)$ we obtain the integral

$$
\int_{0}^{\infty}\{u(t) \cos t x+v(t) \sin t x\} d t .
$$

This will be recognized as an integral having close analogies with Fourier series.t

The object of this paper is to develop the first fundamental convergence properties of the general integrals $J(x)$. In Section 1 I show that the region of convergence of $J(x)$ is a half-plane and determine the orientation of the line which bounds this half-plane. In Section 2 the corresponding results

* All integrals involved in the paper are taken in the sense of Cauchy-Riemann.

$\dagger$ This integral has been treated by many writers. For the principal references up to 1912 see Encyclopédie des Sciences Mathématiques, II, 5, pp. 35-41. Among later papers may be mentioned the following: Pincherle, A c t a M a the matic a, vol. 36 (1913), pp. 269280; Horn, Crelle's J o u rn a l, vol. 144 (1914), pp. 167-189, and vol. 146 (1915), pp. 95-115; Horn, Jahresbericht der Deutschen M thematiker-Vere i n i g u g, vol. 24 (1916), pp. 309-329, and vol. 25 (1916), pp. 74-83.

$\ddagger$ In a similar way a Fourier series may be obtained as a sum of two series $\Omega(x)$. 
for the region of absolute convergence are stated. The boundary of the halfplane of absolute convergence has the same orientation as the boundary of the half-plane of convergence. In Section 3 I consider uniform convergence in closed regions and the consequent properties as to analyticity of functions defined by integrals $J(x)$. Finally, in Section 4 I determine the position of the lines of convergence and absolute convergence in terms of $\phi$ and $g$. The formulæ are analogous to those by which the radius of convergence of a power series is determined in terms of the coefficients. Throughout the paper I have freely used the methods by which Landauł has treated a special case of the integrals $J(x)$.

\section{Character of the region of convergence of $J(x)$}

A point $\rho$ in the complex $x$-plane corresponding to which there exists a nonnegative real value $t_{1}$ of $t$ such that the function $z\left(x+t_{1}\right) / g(x)$ of $x$ has a singularity at $x=\rho$ will be called an exceptional point for the integral $J(x)$. All other points will be called non-exceptional points. We shall also speak of $x=\rho$ as an exceptional or non-exceptional number or value in the respective cases.

Let $x_{0}$ and $x_{1}$ be two values of $x$ which are non-exceptional for the integral $J(x)$ and suppose that $J\left(x_{0}\right)$ converges. We seek conditions under which $J\left(x_{1}\right)$ certainly converges.

Let us write

$$
G(\tau)=\int_{a}^{\tau} \phi(t) g\left(x_{0}+t\right) d t
$$

where the non-ne gative real constant $\alpha$ is chosen so that $g\left(x_{0}+t\right)$ is different from zero for every $t \geqq \alpha$ and where $\tau \geqq \alpha$. Since $J\left(x_{0}\right)$ converges it is clear thăt $G(\tau)$ approaches a finite limit as $\tau$ becomes infinite. Consider the integral

$$
U(\tau)=\int_{a}^{\tau} \phi(t) g\left(x_{1}+t\right) d t=\int_{a}^{\tau} \phi(t) g\left(x_{0}+t\right) \frac{g\left(x_{1}-t\right)}{g\left(x_{0}+t\right)} d t .
$$

To prove that $J\left(x_{1}\right)$ converges it is clearly sufficient to show hat $U(\tau)$ approaches a finite limit as $\tau$ becomes infinite. Integrating by parts in (6) we have the following formula (which we shall need later):

$$
U(\tau)=G(\tau) \frac{g\left(x_{1}+\tau\right)}{g\left(x_{0}+\tau\right)}-\int_{a}^{\tau} G(t)\left(\frac{d}{d t} \frac{g\left(x_{1}+t\right)}{g\left(x_{0}+t\right)}\right) d t .
$$

This brings us to a consideration of the asymptotic character of $g(\xi+t)$ with respect to $t$ for the fixed value $\xi$ of $x$. It is easy to see that we have a

†Sitzungsberichtedermathematisch-physikalischen Klasse der Königlichen Bayerischen Akademieder Wissenschaftenzu M ü n ch e n, vol. 36 (1906), pp. 151-218. See especially pp. 208-218. 
relation of the form

$$
g(\xi+t) \sim e^{p(t, \xi) \log \tau+q(t, \xi)}\left(1+\frac{c_{1}}{t}+\frac{c_{2}}{t^{2}}+\cdots\right)
$$

where the $c$ 's are independent of $t$ and where $p(t, \xi)$ and $q(t, \xi)$ are quantities which may be written in the form

$$
\begin{aligned}
p(t, \xi) & =\nu_{0}(t)+\nu_{1}(t) \xi+\nu_{2}(t) \xi^{2}+\cdots+\nu_{k}(t) \xi^{k} \\
q(t, \xi) & =\beta_{0}(t)+\beta_{1}(t) \xi+\beta_{2}(t) \xi^{2}+\cdots+\beta_{m}(t) \xi^{m} \\
& +\gamma_{1}(t) \xi+\gamma_{2}(t) \xi^{2}+\cdots+\gamma_{k}(t) \xi^{k}
\end{aligned}
$$

Here the quantities $\nu, \beta, \gamma$ have the values

$$
\begin{aligned}
& \nu_{s}(t)=\sum_{i=s}^{k}\left(\begin{array}{l}
i \\
s
\end{array}\right) \mu_{i} t^{i-s}, s=0,1, \cdots, k ; \\
& \beta_{s}(t)=\sum_{i=s}^{m}\left(\begin{array}{c}
i \\
s
\end{array}\right) \alpha_{i} t^{i-s}, s=0,1, \cdots, m ; \\
& \gamma_{s}(t)=\sum_{i=0}^{k} \mu_{i} t^{i-s}\left[\left(\begin{array}{c}
i \\
s-1
\end{array}\right)-\frac{1}{2}\left(\begin{array}{c}
i \\
s-2
\end{array}\right)+\frac{1}{3}\left(\begin{array}{c}
i \\
s-3
\end{array}\right)-\cdots\right], \\
& s=1,2, \cdots, k
\end{aligned}
$$

From (8) it follows that we have with respect to $t$ the asymptotic relation

$$
\frac{g\left(x_{1}+t\right)}{g\left(x_{0}+t\right)} \sim e^{\log t\left\{p\left(t, x_{1}\right)-p\left(t, x_{0}\right)\right\}+\left\{q\left(t, \dot{x}_{1}\right)-q\left(t, x_{0}\right)\right\}}\left(1+\frac{d_{1}}{t}+\frac{d_{2}}{t^{2}}+\cdots\right),
$$

where the $d$ 's are independent of $t$. We see that $p\left(t, x_{1}\right)-p\left(t, x_{0}\right)$ is a polynomial in $t$ of degree $k-1$ and that $q\left(t, x_{1}\right)-q\left(t, x_{0}\right)$ is a polynomial in $t$ and that its degree is $m-1$ in case $m$ is greater than $k$. (If $k$ is zero the corresponding difference function is zero.)

By differentiating we obtain from (11) the asymptotic relation*

$$
\frac{d}{d t} \frac{g\left(x_{1}+t\right)}{g\left(x_{0}+t\right)} \sim e^{r(t)}\left(-\frac{d_{1}}{t^{2}}-\frac{2 d_{2}}{t^{1}}-\cdots\right)+e^{r(t)} r^{\prime}(t)\left(1+\frac{d_{1}}{t}+\cdots\right),
$$

where $r(t)$ denotes the exponent on $e$ in (11) and $r^{\prime}(t)=d r / d t$, the relation (12) being valid in the interior of $V$.

From (7) and (12) we see that

$$
U(\tau)=G(\tau) \frac{g\left(x_{1}+\tau\right)}{g\left(x_{0}+\tau\right)}-\int_{a}^{\tau} G(t) T(t) d t-\int_{a}^{\tau} G(t) S(t) d t
$$

where $T(t)$ and $S(t)$ are suitable functions asymptotic to the first and second terms, respectively, in the second member of (12).

* For a theorem justifying this differentiation see a paper by J. F. Ritt in B u ll e $t$ i n of the American Mathematical Societ y, vol. 24 (1918), pp. 225-227. 
It is convenient to make a separation of cases. We suppose first that $k<m$. Then $m \geqq 2$, since by hypothesis $m>1$ if $k=0$. The dominant term in the exponent of the second member of (11), when this exponent is expressed as a polynomial in $t$ and $\log t$, is

$$
m \alpha_{m}\left(x_{1}-x_{0}\right) t^{m-1} \text {. }
$$

Now a constant $A$ exists such that $|G(t)|<A$ for every real value of $t$ not less than $\alpha$. Hence if $R\left\{\alpha_{m}\left(x_{1}-x_{0}\right)\right\}^{*}$ is negative, it is clear that each term in the second member of (13) approaches a finite limit as $\tau$ becomes infinite; and thence that $J\left(x_{1}\right)$ is convergent.

In the next place suppose that $k \geqq m$ and $k>1$. Precisely similar considerations lead to the conclusion that $J\left(x_{1}\right)$ is convergent in case $R\left\{\mu_{k}\left(x_{1}-x_{0}\right)\right\}$ is negative.

There remains the case in which $k=1$ and $m=0$ or 1 . We shall show in this case that $J\left(x_{1}\right)$ is convergent provided that $R\left\{\mu_{1}\left(x_{1}-x_{0}\right)\right\}$ is negative. For this special case (8) takes the form

$$
g(\xi+t) \sim t^{\mu_{0}+\mu_{1}(\xi+t)} e^{a_{0}+\mu_{1} \xi+\beta(\xi+t)}\left(1+\frac{c_{1}}{t}+\cdots\right),
$$

where $\beta=\alpha_{1}$ or 0 according as $m=1$ or 0 . Then

$$
\frac{g\left(x_{1}+t\right)}{g\left(x_{0}+t\right)} \sim t^{\mu_{1}\left(x_{1}-x_{0}\right)} e^{\left(\mu_{1}+\beta\right)\left(x_{1}-x_{0}\right)}\left(1+\frac{d_{1}}{t}+\cdots\right) .
$$

From this we see readily that the integrals

$$
\int_{a}^{\infty} G(t) T(t) d t, \quad \int_{a}^{\infty} G(t) S(t) d t
$$

converge. Hence $U(\tau)$ approaches a finite limit as $\tau$ becomes infinite and $J\left(x_{1}\right)$ is therefore convergent.

Hence we have the following theorem (cf. Theorem I of Memoir I).

Theorem I. Let $x_{0}$ and $x_{1}$ be two values of $x$ which are non-exceptional for the integral $J(x)$ and suppose that $J\left(x_{0}\right)$ converges. Then $J\left(x_{1}\right)$ also converges provided $R\left(\sigma x_{1}\right)<R\left(\sigma x_{0}\right)$, where $\sigma$ denotes $\alpha_{m}$ or $\mu_{k}$ according as $m$ is or is not greater than $k$.

By a region $C$ of convergence of the integral $J(x)$ we shall mean a region such that $J(x)$ converges for every non-exceptional value of $x$ in the interior of $C$ and diverges for every non-exceptional value of $x$ exterior to $C$. In a similar way we define a region $\Gamma$ of absolute convergence.

By means of Theorem I it is easy to determine the character of the region of convergence of $J(x)$. Compare the related argument in Memoir I, p. 214. We have the following result.

\footnotetext{
* We employ the symbol $R(z)$ to denote the real part of $z$.
} 
Theorem II. There exists a real number $\lambda$ such that the region of convergence of $J(x)$ is bounded by the straight line $R(\sigma x)=\lambda$ and lies on that side of this line for which $R(\sigma x)<\lambda$.

This result may be compared with Theorem II of Memoir I. As to convergence on the boundary of the region of convergence one may establish properties similar to those given for $\Omega(x)$ in Memoir I, pp. 214-216, the method of treatment for $J(x)$ being analogous to that for $\Omega(x)$.

\section{Character of the Region of absolute convergence}

In view of (11) we have from (6), by means of an obvious argument, the following theorems.

Theorem III. If $x_{0}$ and $x_{1}$ are two values of $x$ which are non-exceptional for $J(x)$ and if $J\left(x_{0}\right)$ converges absolutely, then $J\left(x_{1}\right)$ converges absolutely provided that $R\left(\sigma x_{1}\right)<R\left(\sigma x_{0}\right)$.

A ready consequence of this is the following theorem.

THEOREM IV. There exists a real number $\mu$ such that the region of absolute convergence of the integral $J(x)$ is bounded by the straight line $R(\sigma x)=\mu$ and lies on that side of this line for which $R(\sigma x)<\mu$.

\section{UNIFORM CONVERGENCE}

We shall now prove the following theorem (compare Theorem III of Memoir I).

Theorem V. The integral $J(x)$ converges uniformly in any closed domain $D$ which lies within its region of convergence and contains no point which is exceptional for $J(x)$ or is a limit point of points which are exceptional for $J(x)$.

From the character of $D$ as defined in the theorem it is clear that a nonexceptional point $x_{0}$ and a positive constant $\epsilon$ exist such that $J\left(x_{0}\right)$ converges and $R\left(\sigma x_{0}\right)$ is less than $R\left(\sigma x_{1}\right)-\epsilon$ for every $x_{1}$ in $D$. Let us now denote the integral $U(\tau)$ of $(6)$ by $U\left(x_{1}, \tau\right)$ in order to put in evidence the variable $x_{1}$ which is to range over $D$. To prove the theorem it is sufficient to show that

$$
\lim _{\tau=\infty} U\left(x_{1}, \tau\right)
$$

exists uniformly as to $x_{1}$ ranging over $D$. The changes (mostly verbal in character) which may be made in the proof of Theorem I in order to reach this conclusion are now obvious. The argument need not be given in detail.

As an immediate consequence of Theorem $\mathrm{V}$ we have the following theorem.

Theorem VI. The integral $J(x)$ defines a function $J(x)$ of $x$ which is analytic at every non-exceptional point which lies in the interior of its region of convergence and is not a limit point of exceptional points, and the derivatives of $J(x)$ at every such point may be found by differentiating the integral $J(x)$ under the sign of integration. 


\section{Convergence Numbers For the INTEgRaL $J(x)$}

For the determination of the convergence numbers we have the following theorem which we shall now demonstrate (compare Theorem XII and corollary of Memoir I).

Theorem VII. The convergence [absolute convergence] number $\lambda[\mu]$ for the integral $J(x)$ is expressed in terms of $\phi$ and $g$ by the following relations:

(1) in case $k<m$ we have

$$
\lambda=-\limsup _{u=\infty} \frac{\log \left|\int_{u}^{u+1} \phi(t) g(t) d t\right|}{m u^{m-1}}\left[\mu=-\limsup _{u=\infty} \frac{\log \int_{u}^{u+1}|\phi(t) g(t)| d t}{m u^{m-1}}\right] ;
$$

(2) in case $k \geqq m$ and $k>1$ we have

$$
\lambda=-\limsup _{u=\infty} \frac{\log \left|\int_{u}^{u+1} \phi(t) g(t) d t\right|}{k u^{k-1} \log u}\left[\mu=-\limsup _{u=\infty} \frac{\log \int_{u}^{u+1}|\phi(t) g(t)| d t}{k u^{k-1} \log u}\right] ;
$$

(3) in case $k=1$ and $m=0$ or 1 we have

$$
\lambda=-\limsup _{u=\infty} \frac{\log \left|\int_{e E(u)}^{e^{u}} \phi(t) g(t) d t\right|}{u}\left[\mu=-\limsup _{u=\infty} \frac{\log \int_{e B(u)}^{e u}|\phi(t) g(t)| d t}{u}\right],
$$

where $E(u)$ denotes the integral part of $u$.

Let us first prove the formulas for $\mu$. Taking up case (1), let us write

$$
\limsup _{u=\infty} \frac{\log \int_{u}^{u+1}|\phi(t) g(t)| d t}{m u^{m-1}}=\zeta
$$

In the first place we take $\zeta$ to be finite. Then for every positive constant $\delta / 2$ there exists a $U$ such that

$$
\int_{u}^{u+1}|\phi(t) g(t)| d t<e^{m u^{m-1}(\zeta+\delta / 2)}
$$

provided that $u \geqq U$. To show that $J\left(x_{1}\right)$ converges absolutely it is therefore sufficient to prove the convergence of the series

$$
\sum_{n=0}^{\infty} M_{n} e^{m(U+n)^{m-1}(\zeta+\delta / 2)},
$$

where $M_{n}$ is the least upper bound of the values of $\left|g\left(x_{1}+t\right) / g(t)\right|$ in the interval $U+n \leqq t \leqq U+n+1$. We take $x_{1}$ a non-exceptional point such that $R\left(\alpha_{m} x_{1}\right)=-\zeta-\delta$. Employing (11) with $x_{0}$ replaced by 0 we conclude readily that the foregoing infinite series is convergent. Hence $J\left(x_{1}\right)$ 
converges absolutely when $x_{1}$ is a non-exceptional point such that $R\left(\alpha_{m} x_{1}\right)$ $<-\zeta$. Hence $\mu \nless-\zeta$.

To prove that $\mu \ngtr-\zeta$ we proceed as follows. For every positive constant $\delta / 2$ there exists an infinite sequence of numbers $u_{1}, u_{2}, u_{3}, \cdots$ such that $u_{i+1}-u_{i} \geqq 1$ and

$$
\int_{u}^{u+1}|\phi(t) g(t)| d t>e^{m u^{m-1}(\zeta-\delta / 2)}
$$

for $u=u_{1}, u_{2}, u_{3}, \cdots$. Now let $x_{1}$ be a non-exceptional point for $J(x)$ such that $R\left(\alpha_{m} x_{1}\right)=-\zeta+\delta$. Then the value of the integral

$$
I_{i}=\int_{u_{i}}^{u_{i}+1}\left|\phi(t) g(t) \frac{g\left(x_{1}+t\right)}{g(t)}\right| d t
$$

clearly does not approach zero as $i$ increases indefinitely, as one sees by aid of relation (11); whence it follows that $J\left(x_{1}\right)$ is not absolutely convergent. Hence $\mu \ngtr-\zeta$.

In case $\zeta$ is infinite it may be shown that for every $M$ and positive $\delta$ a set of numbers $u_{1}, u_{2}, u_{3}, \cdots$ exists such that $u_{i+1}-u_{i} \geqq 1$ and

$$
\int_{u_{i}}^{u_{i}+1}|\phi(t) g(t)| d t>e^{m u_{i} m-1(M+\delta)} .
$$

Then if $x_{1}$ is a non-exceptional value for $J(x)$ such that $R\left(\alpha_{m} x_{1}\right)=-M$, it is clear that the value of the integral $I_{i}$ does not approach zero as $i$ increases. Hence when $\zeta$ is infinite the integral $J(x)$ converges absolutely for no nonexceptional value of $x$.

We conclude therefore to the validity of the formula for $\mu$ in case (1).

The determination of the value of $\mu$ in cases (2) and (3) is so closely parallel to that employed in case (1) that it is unnecessary to give a statement of it.

Proceeding to establish the formula for $\lambda$ in case (1), let us write

$$
\limsup _{u=\infty} \frac{\log \left|\int_{u}^{u+1} \phi(t) g(t) d t\right|}{m u^{m-1}}=\zeta .
$$

First let $\zeta$ be finite. Then for every positive constant $\delta / 2$ there exists a $U$ such that

$$
\left|\int_{u}^{u+1} \phi(t) g(t) d t\right|<e^{m u m-1(\zeta+\delta / 2)} \quad \text { when } \quad u \geqq U .
$$

Let $x_{1}$ be a non-exceptional point such that $R\left(\alpha_{m} x_{1}\right)=-\zeta-\delta$ and consider the second member of (13) for $\tau$ becoming infinite, $x_{0}$ now having the value zero. Through use of (11) it is easy to see that its first term approaches a finite limit as $\tau$ increases indefinitely. That each of the other terms does 
so also may be shown readily through use of (12). Hence $J\left(x_{1}\right)$ converges and $\lambda \nless-\zeta$.

To prove that $\lambda \ngtr-\zeta$ we let $x_{0}$ be a non-exceptional point for which $J\left(x_{0}\right)$ converges and prove that for every positive constant $\delta$ there exists a $U$ such that

$$
\left|\int_{u}^{u+1} \phi(t) g(t) d t\right|<e^{m u m-1(\xi+\delta)} \quad \text { for } \quad u \geqq U,
$$

where $\xi=-R\left(\alpha_{m} x_{0}\right)$.

If in the computation by which (13) was derived we write $u$ and $u+1$ for $\alpha$ and $\tau$, respectively, and take $x_{1}=0$, we have

$$
\begin{aligned}
&\left.\left|\int_{u}^{u+1} \phi(t) g(t) d t\right| \leqq \mid G(\tau) \frac{g(\tau)}{g\left(x_{0}+\tau\right)}\right]_{u}^{u+1}\left|+\int_{u}^{u+1}\right| G(t) T(t) \mid d t \\
&+\int_{u}^{u+1}|G(t) S(t)| d t .
\end{aligned}
$$

From the convergence of $J\left(x_{0}\right)$ it follows that a constant $B$ exists such that $|G(t)|<B$ when $t \geqq \alpha$. Through (11) and (12) one now concludes readily to the truth of $(16)$.

Hence the formula for $\lambda$ is valid in case (1) if $\zeta$ is finite. Moreover, the argument just ended shows that $\zeta$ can be infinite only when $J(x)$ diverges for every non-exceptional value $x_{0}$. Hence the formula for $\lambda$ is valid in case (1) when $\zeta$ is infinite.

Case (2) can be dealt with in the same manner as case (1).

In case (3) let us write

$$
\lim _{n=\infty} u^{-1} \log \left|\int_{e^{K(u)}}^{e^{u}} \phi(t) g(t) d t\right|=\zeta .
$$

Suppose first that $\zeta$ is finite. Then for every positive constant $\delta / 2$ a constant $U$ exists such that

$$
\left|\int_{e^{E(u)}}^{e^{u}} \phi(t) g(t) d t\right|<e^{u(\zeta+\delta / 2)} \quad \text { when } \quad u \geqq U .
$$

Let $x_{1}$ be a non-exceptional point for $J(x)$ such that $R\left(\mu_{1} x_{1}\right)=-\zeta-\delta$. We shall prove that $J\left(x_{1}\right)$ converges.

We shall employ relation (13) with $x_{0}$ replaced by $0, \alpha$ being chosen so that $\alpha=e^{k}$ where $k$ is a positive integer not less than $E(U)$ and so that $g(t)$ is different from zero for $t \geqq \alpha$. To prove the convergence of $J\left(x_{1}\right)$ it is sufficient to show that $\lim _{\tau=\infty} U(\tau)$ exists and is finite, $U(\tau)$ being defined as in (6).

We have

$$
\begin{aligned}
G(\tau) & =\int_{a}^{\tau} \phi(t) g(t) d t \\
& =\sum_{l=1}^{l} \int_{e^{k+i-1}}^{e^{k+i}} \phi(t) g(t) d t+\int_{e^{k+l}}^{\tau} \phi(t) g(t) d t,
\end{aligned}
$$


where $k+l$ is the greatest integer such that $e^{k+l}<\tau$. Hence

$$
|G(\tau)|<\sum_{i=1}^{l} e^{(k+i)(\zeta+\delta / 2)}+e^{\log \tau \cdot(\zeta+\delta / 2)}<\log \tau \cdot \tau^{\zeta+\delta / 2} .
$$

Since $R\left(\mu_{1} x_{1}\right)=-\zeta-\delta$ it is now easy to see from (13) by aid of (14) and (12) that $\lim _{\tau=\infty} U(\tau)$ exists and is finite.

From this it follows that $J\left(x_{1}\right)$ converges. Hence we conclude that $\lambda \nless-\zeta$ when $\zeta$ is finite.

Let us suppose next that $x_{0}$ is a non-exceptional point for which $J\left(x_{0}\right)$ converges. To complete the proof for $\lambda$ in case (3) when $\zeta$ is finite it is sufficient to show that for every positive constant $\delta$ there exists a $U$ such that

$$
\left|\int_{e^{E(u)}}^{e^{u}} \phi(t) g(t) d t\right|<e^{u(\xi+\delta)} \quad \text { for } \quad u \geqq U,
$$

where $\xi=-R\left(\mu_{1} x_{0}\right)$.

If in the computation by which (13) was derived we write $e^{E(u)}$ for $\alpha$, $e^{u}$ for $\tau$, and take $x_{1}=0$, we have

$$
\begin{aligned}
\left.\left|\int_{\epsilon^{E(u)}}^{e^{u}} \phi(t) g(t) d t\right| \leqq \mid G(\tau) \frac{g(\tau)}{g\left(x_{0}+\tau\right)}\right]_{\tau=e^{E(u)}}^{\tau=e^{u}}\left|+\int_{e^{E(u)}}^{e^{u}}\right| G(t) T(t) \mid d t & +\int_{e^{E(u)}}^{e^{u}}|G(t) S(t)| d t .
\end{aligned}
$$

Since $J\left(x_{0}\right)$ converges it is clear that a constant $B$ exists such that $|G(t)|<B$. Hence, from the last preceding inequality, by aid of (14) and (12), we see readily that (17) is a valid relation. Hence $\lambda \ngtr-\zeta$ when $\zeta$ is finite.

The argument just ended shows that $\zeta$ can be infinite only when $J(x)$ diverges for every non-exceptional value $x_{0}$. Hence the formula for $\lambda$ in case (3) is valid whether $\zeta$ is finite or infinite.

This completes the proof of the theorem.

University of IllinoIs 